\title{
Molecular profiling of immunoglobulin heavy- chain gene rearrangements unveils new potential prognostic markers for multiple myeloma patients
}

Alejandro Medina (1]', Cristina Jiménez', M. Eugenia Sarasquete, Marcos González', M. Carmen Chillón', Ana Balanzategui ', Isabel Prieto-Conde (1)', María García-Álvarez', Noemí Puig', Verónica González-Calle', Miguel Alcoceba (1)', Isabel Cuenca², Santiago Barrio², Fernando Escalante ${ }^{3}$, Norma C. Gutiérrez ${ }^{1}$, Mercedes Gironella ${ }^{4}$, Miguel T. Hernández ${ }^{5}$, Anna Sureda ${ }^{6}$, Albert Oriol $^{7}$, Joan Bladé ${ }^{8}$, Juan-José Lahuerta ${ }^{2}$, Jesús F. San Miguel ${ }^{9}$,

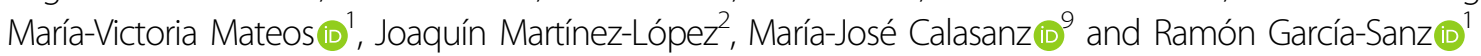

\begin{abstract}
Multiple myeloma is a heterogeneous disease whose pathogenesis has not been completely elucidated. Although B-cell receptors play a crucial role in myeloma pathogenesis, the impact of clonal immunoglobulin heavy-chain features in the outcome has not been extensively explored. Here we present the characterization of complete heavychain gene rearrangements in 413 myeloma patients treated in Spanish trials, including 113 patients characterized by next-generation sequencing. Compared to the normal B-cell repertoire, gene selection was biased in myeloma, with significant overrepresentation of IGHV3, IGHD2 and IGHD3, as well as IGHJ4 gene groups. Hypermutation was high in our patients (median: 8.8\%). Interestingly, regarding patients who are not candidates for transplantation, a high hypermutation rate $(\geq 7 \%)$ and the use of IGHD2 and IGHD3 groups were associated with improved prognostic features and longer survival rates in the univariate analyses. Multivariate analysis revealed prolonged progression-free survival rates for patients using IGHD2/IGHD3 groups (HR: $0.552,95 \% \mathrm{Cl}: 0.361-0.845, p=0.006$ ), as well as prolonged overall survival rates for patients with hypermutation $\geq 7 \%$ (HR: $0.291,95 \% \mathrm{Cl}: 0.137-0.618, p=0.001$ ). Our results provide new insights into the molecular characterization of multiple myeloma, highlighting the need to evaluate some of these clonal rearrangement characteristics as new potential prognostic markers.
\end{abstract}

\section{Introduction}

B-cell lymphoproliferative disorders are caused by the expansion of a pathological clone at a specific stage of differentiation reflected in the B-cell receptor (BCR). Through multiple, hierarchically structured events, BCR genes from each $B$ cell are assembled in a particular shape that confers an extremely high diversity to the B-cell repertoire to recognize different antigens ${ }^{1}$. IGHV, IGHD

\footnotetext{
Correspondence: M. Eugenia Sarasquete (mealonsos@saludcastillayleon.es) 'Hospital Universitario de Salamanca (HUSAL), IBSAL, IBMCC (USAL-CSIC), CIBERONC, Salamanca, Spain

${ }^{2}$ Hospital 12 de Octubre, CIBERONC, Madrid, Spain

Full list of author information is available at the end of the article
}

and IGHJ immunoglobulin genes go through recombination during early B-cell ontogeny that takes place in the bone marrow (BM), an entirely random, antigenindependent process driven by the RecombinationActivating Genes (RAG1 and RAG2) ${ }^{2}$ and Terminal deoxynucleotidyl Transferase $(T d T)^{3}$. In humans, there are more than $50 I G H V$ genes, 27 IGHD genes and 6 IGHJ genes. $I G H V$ and $I G H D$ genes are clustered into seven different groups ${ }^{4}$. During B-cell differentiation, one allele is rearranged to produce a complete heavy-chain rearrangement (IGH) with or without the rearrangement of the second $I G H$ allele ${ }^{5}$. This means that one or two IGH rearrangements can be found in each individual B cell, 
although only one of them is expressed as a functional protein ${ }^{6}$. Immunoglobulin-expressing B cells then migrate to germinal centers to undergo two antigen-dependent processes: somatic hypermutation (SHM) and classswitch recombination $(\mathrm{CSR})^{7}$.

As a clonal B-cell malignancy, multiple myeloma (MM) arises from a single cell, that has been recognized as a post-germinal center, IgA/IgG switched B cell in several studies $^{8}$. VDJH usage, Complementarity-determining Region 3 (CDR3) composition and somatic hypermutation (SHM) levels have been studied for the vast majority of B-cell malignancies, as well as for normal, healthy B cells $^{9-12}$. The most interesting finding to date is the close association between SHM rate and the outcome in chronic lymphocytic leukemia (CLL), since a high SHM rate $(>2 \%)$ is associated with good prognosis ${ }^{13}$. Another interesting observation is the presence of stereotyped immunoglobulin receptors, not only in $\mathrm{CLL}^{14}$ but also in mantle-cell lymphoma (MCL) or marginal zone lymphoma (MZL), which led researchers to infer that this was a common phenomenon for all mature B-cell tumors with potential prognostic and therapeutic implications ${ }^{15}$. Several studies performed with MM patients treated with conventional chemotherapy did not find any correlation between prognosis and $V D J H$ gene usage, CDR3 amino acid composition or SHM rates ${ }^{16-18}$. However, most series often included an insufficient number of heterogeneously treated patients, which makes any conclusion difficult to be inferred.

Here, we present the largest-to-date analysis of the $V D J H$ repertoire in multiple myeloma, consisting in biological and clinical data from 413 patients diagnosed and treated according to the Spanish Myeloma Group (GEMPETHEMA) protocols. Data were used for a comprehensive investigation of $V D J H$ rearrangement characteristics, including IGHV, IGHD and IGHJ gene usage, SHM level and distribution, or complementarity-determining region (CDRs) and framework region (FWRs) length and composition. We also tried to identify the presence of potential clusters of stereotyped receptors, assessing potential relationships between molecular characteristics and clinical outcomes.

\section{Methods \\ Patients}

A total of 413 newly diagnosed MM patients, diagnosed from 1995 to 2016, were included in the present study. Most of them, 319 (77\%), were enrolled in clinical trials from the GEM-PETHEMA Spanish MM group: GEM2000 ${ }^{19}$ (NCT00560053, $n=27$ ), GEM2005más65 ${ }^{20}$ (NCT00443235, $\left.n=23\right)$, GEM2005MENOS65 $^{21}$ (NCT00461747, $n=20$ ), GEM2010MAS65 ${ }^{22}$ (NCT01237249, $n=83)$, QUIREDEX ${ }^{23}$ (NCT00480363, $n=29$ ), GEM2012MENOS65 $^{24}($ NCT01916252, $n=130)$ and GEM-CESAR ${ }^{25}$
(NCT00480363, $n=7$ ). The 94 remaining patients were treated following the same protocols (GEM2000, GEM2005), but they were not formally included in any clinical trial.

According to the International Myeloma Working Group (IMWG) criteria $^{26}$, patients with either $t(4 ; 14)$, $t(14 ; 16)$ or del17p were grouped together as a high-risk (HR) cytogenetics subset for subsequent analysis. Patients with symptomatic myeloma were also stratified based on the Revised International Staging System (R-ISS) ${ }^{27}$.

This study was approved by the Ethical Committee of the University Hospital of Salamanca in accordance with the Spanish law and the Declaration of Helsinki principles. Written informed consent was obtained from every patient prior to the inclusion in each trial.

\section{Sample collection and IGH sequencing}

Genomic DNA was isolated from bone marrow aspirates at the time of diagnosis using the automated DNA Purification kit Maxwell ${ }^{\circledR}$ (Promega, Madison, WI, USA). DNA quantification and quality assessment were done using the NanoDrop2000 Spectrophotometer (ThermoFisher, Waltham, MA, USA). For the amplification of complete $V D J H$ rearrangements, we used the BIOMED-2 (now Euroclonality $\odot$ ) FR1 primers ${ }^{28}$ in multiplexed PCR reactions. All reactions were carried out in a $25 \mu \mathrm{L}$ mixture containing $\sim 100 \mathrm{ng}$ DNA and $10 \mu \mathrm{mol}$ of each primer. Monoclonal assessment of amplified products was carried out by GeneScanning using $1 \mu \mathrm{L}$ of PCR reaction. PCR products were sequenced in an automated ABI3500 XL DNA sequencer using Big-Dye terminators v3.1 (Applied Biosystems ${ }^{\mathrm{TM}}$, Foster City, CA, USA).

One hundred and thirteen samples were also analyzed by next-generation sequencing (NGS) using the LymphoTrack ${ }^{\circledast}$ methodology (Invivoscribe Technologies, San Diego, CA, USA). We targeted the Framework Region 1 to amplify VDJH rearrangements from 113 patients. In one-step PCR, amplicons were generated and indexed. A purification step using Agentcourt AMPure XP microbeads (Beckman Coulter Inc, Brea, CA, USA) was performed; then, we assessed the quality of our amplicons using the TapeStation 4200 (Agilent, Santa Clara, CA, USA) and Qubit 2.0 (ThermoFisher, Waltham, MA, USA). Libraries were sequenced in an MiSeq platform (Illumina, San Diego, CA, USA) with $2 \times 251$ of read length and aiming for 1 million reads per sample.

\section{Immunophenotypic characterization}

Patients were routinely characterized at diagnosis by multiparametric flow cytometry. Bone marrow samples were processed within $48 \mathrm{~h}$ after collection. Analysis was performed using different assays, following the EuroClonality/EuroFlow consortium guidelines: six-color panel for six markers (CD38/CD138/CD45/CD19/CD56/ 
CD117) in 78 patients, eight-color panel for eight markers (CD38/CD138/CD45/CD19/CD56/CD117/CD27/CD81) in the remaining 335 patients.

For data analysis, the values of all parameters measured per tube were mathematically calculated for the individual plasma-cell events using the merge and calculation functions of the INFINICYT ${ }^{\mathrm{TM}}$ v2.0 software (Cytognos S. L. Salamanca, Spain). Plasma cells were identified based on the characteristic pattern of expression of CD38, CD138, CD45 and light scatter features.

\section{Sequence analysis}

$I G H V, I G H D$ and IGHJ genes from complete rearrangements detected using either Sanger or NGS were identified comparing them with the IMGT/V-Quest database $^{29}$, taking into account the option for insertions/ deletions. Mutational status was assessed using the closest germline gene. Sequences containing more than $2 \%$ deviation from the germline were considered somatically mutated, following previously described criteria ${ }^{13}$. Point mutations were annotated, as well as the length and composition of N-, P- and CDR3 regions.

FastQ files generated by deep sequencing were processed using the LymphoTrackAnalysis ${ }^{\circledR}$ tool to retrieve sequences from virtually every clonal B cell in the samples, allowing the identification of tumor clones if the following criteria were met: (i) $\geq 20,000$ total reads; (ii) at least one but not more than 2 merged top reads $\geq 2.5 \%$ of total reads and (iii) top first or second merged reads must be at least twice more abundant than the third most frequent read to be considered clonotypic.

Rearrangements were considered biallelic if the first two reads met the aforementioned criteria, had similar read count (less than $50 \%$ difference) and one was productive while the other one unproductive. Moreover, rearrangements were considered potentially biclonal if the first two reads met the aforementioned criteria, had similar read count (less than 50\% difference) and both were productive.

\section{CDR3 clustering analysis}

ClustalX2.0 was used as previously described ${ }^{30,31}$, comparing CDR3 amino acid sequences obtained from our cohort and 1117 additional sequences obtained from the IMGT/LIGM-DB database ${ }^{32}$ corresponding to unique rearrangements from both normal and human tumor B cells.

Primary and secondary standard criteria ${ }^{31,33}$ were used as described before to characterize immunoglobulin clusters.

\section{Statistical analysis}

Clinical and biological characteristics were analyzed discriminating between transplant-eligible and noneligible patients, as well as asymptomatic patients, with the SPSS
20.0 software (IBM, Armonk, NY, USA) using Fisher's exact test for discrete variables and the Mann-Whitney test for continuous variables. The Kaplan-Meier method and the log-rank test were used to plot and compare progression-free survival (PFS) and overall survival (OS) curves. Cox regression was used to perform the multivariate analysis. Receiver operating characteristic (ROC) curves were used to evaluate potential cutoff values for some variables. PFS was defined as the time from diagnosis to either disease progression, death or the last follow-up visit. OS was defined as the time from diagnosis to the last follow-up visit or decease. All reported $p$ values were obtained by a two-sided exact method, at the conventional $5 \%$ significance level $(p<0.05)$.

\section{Results}

\section{Clinical characteristics}

Baseline characteristics of the 413 patients included in the study are summarized in Table 1.

For transplant-eligible patients $(n=228)$, R-ISS stages I, II and III represented $24.1 \%, 62.8 \%$ and $13.1 \%$ of this subset, respectively. After induction, before the pretransplant conditioning regimen started, the overall response ratio (ORR) was 92.4\%: $15.3 \%$ were in stringent complete response (sCR); $24.1 \%$ of patients had achieved complete response (CR); and $26.5 \%$ of patients were in very good partial response (VGPR). The remaining patients were in partial response (PR, 26.5\%). Median PFS was 49.5 months; median OS was 67.9 months.

For transplant-ineligible patients $(n=149)$, R-ISS stages I, II and III represented $13.1 \%, 77.9 \%$ and $9 \%$ of this subgroup, respectively. After induction, the ORR was 89.7\%: $7.7 \%$ were in sCR, $34.2 \%$ achieved CR, $14.5 \%$ were in VGPR and $33.3 \%$ were in PR. Median PFS was 26.4 months; median OS was 57.7 months.

As expected, high-risk asymptomatic patients $(n=36)$ were younger (median age: 60 years). Serum albumin levels were higher (median: $3.76 \mathrm{~g} / \mathrm{dL}$ ) and $\beta 2$ microglobulin levels were lower (median: $2.25 \mathrm{mg} / \mathrm{L}$ ). Consequently, $61.9 \%$ of smoldering patients were at ISS stage I, and $38.1 \%$ were at stage II.

\section{Detection of clonal VDJH gene rearrangements}

Sanger sequencing allowed the identification of full clonal $V D J H$ gene rearrangements in $88 \%$ of patients (363/413). In 23 cases, the clonal IGH gene rearrangement was not detected, possibly due to a high polyclonal background or lack of PCR amplification due to an incorrect primer-annealing. In 12 cases, we could only identify the $I G H V$ gene, while in the remaining cases we could identify two out of three genes (mostly $I G H V$ and $I G H J$, but not IGHD, $n=13$ ).

Combining Sanger and NGS, the overall VDJH detection rate was $92.5 \%(382 / 413)$. In all, 388 complete 
Table 1 Patient characteristics.

\begin{tabular}{|c|c|c|c|c|}
\hline Variable & $\begin{array}{l}\text { Global series } \\
(N=413)\end{array}$ & $\begin{array}{l}\text { Transplant-eligible } \\
(N=228)\end{array}$ & $\begin{array}{l}\text { Transplant-ineligible } \\
(N=149)\end{array}$ & $\begin{array}{l}\text { Asymptomatic } \\
(N=36)\end{array}$ \\
\hline \multicolumn{5}{|l|}{ Sex } \\
\hline Men & $54.5 \%$ & $62.1 \%$ & $45.8 \%$ & $54.8 \%$ \\
\hline Women & $45.5 \%$ & $37.9 \%$ & $54.2 \%$ & $45.2 \%$ \\
\hline Age & 64 years (37-91) & 59 years $(37-69)$ & 72 years (48-91) & 60 years $(42-77)$ \\
\hline \multicolumn{5}{|l|}{$\lg \mathrm{H}$} \\
\hline $\lg G$ & $59.4 \%$ & $55.8 \%$ & $62.8 \%$ & $42.3 \%$ \\
\hline $\lg A$ & $32.8 \%$ & $35.5 \%$ & $30.4 \%$ & $50 \%$ \\
\hline Bence-Jones & $6.9 \%$ & $7.0 \%$ & $6.8 \%$ & $7.7 \%$ \\
\hline Nonsecretory & $0.9 \%$ & $1.7 \%$ & $0 \%$ & $0 \%$ \\
\hline \multicolumn{5}{|l|}{$\operatorname{lgL}$} \\
\hline Kappa chain & $62.5 \%$ & $63.5 \%$ & $60.7 \%$ & $50 \%$ \\
\hline Lambda chain & $37.5 \%$ & $36.5 \%$ & $39.3 \%$ & $50 \%$ \\
\hline Calcium & $9.63 \pm 1.72$ mg/dL $(0.45-17)$ & $9.50 \pm 1.99$ mg/dL (0.45-17) & $9.78 \pm 1.37$ mg/dL (2.28-14.4) & $9.11 \pm 0.49 \mathrm{mg} / \mathrm{dL}(8-10)$ \\
\hline Creatinine & $1.16 \pm 0.84 \mathrm{mg} / \mathrm{dL}(0.20-6.50)$ & $1.11 \pm 0.76$ mg/dL $(0.20-6.50)$ & $1.21 \pm 0.93 \mathrm{mg} / \mathrm{dL}(0.36-5.90)$ & $0.89 \pm 0.28 \mathrm{mg} / \mathrm{dL}(0.50-1.61)$ \\
\hline Albumin & $3.52 \pm 0.67 \mathrm{~g} / \mathrm{dL}(1.43-5.50)$ & $3.54 \pm 0.73 \mathrm{~g} / \mathrm{dL}(1.70-5.14)$ & $3.51 \pm 0.61 \mathrm{~g} / \mathrm{dL}(1.43-5.50)$ & $3.76 \pm 0.46 \mathrm{~g} / \mathrm{dL}(2.20-4.60)$ \\
\hline$\beta 2$ microglobulin & $5.17 \pm 5.22 \mathrm{mg} / \mathrm{L}(0-62)$ & $5.11 \pm 5.45 \mathrm{mg} / \mathrm{L}(0-62)$ & $5.20 \pm 4.98$ mg/L (0.15-43.40) & $2.25 \pm 1.13 \mathrm{mg} / \mathrm{L}(0.30-4.41)$ \\
\hline Hemoglobin & $10.54 \pm 1.98 \mathrm{~g} / \mathrm{dL}(4.90-15.60)$ & $10.55 \pm 1.99 \mathrm{~g} / \mathrm{dL}(5.50-15.50)$ & $10.56 \pm 1.97 \mathrm{~g} / \mathrm{dL}(4.90-15.60)$ & $12.38 \pm 1.13 \mathrm{~g} / \mathrm{dL}(10.10-14.70)$ \\
\hline \multicolumn{5}{|l|}{ R-ISS $\mathrm{a}^{\mathrm{a}}$} \\
\hline Stage I & $19.1 \%$ & $24.1 \%$ & $13.1 \%$ & $61.9 \%$ \\
\hline Stage II & $69.7 \%$ & $62.8 \%$ & $77.9 \%$ & $38.1 \%$ \\
\hline Stage III & $11.2 \%$ & $13.1 \%$ & $9.0 \%$ & $0 \%$ \\
\hline \multicolumn{5}{|l|}{ ECOG } \\
\hline 0 & $23.8 \%$ & $27.6 \%$ & $20.3 \%$ & $66.7 \%$ \\
\hline 1 & $49.7 \%$ & $49.3 \%$ & $50.7 \%$ & $33.3 \%$ \\
\hline 2 & $20.2 \%$ & $17.8 \%$ & $21.6 \%$ & $0 \%$ \\
\hline 3 & $4.0 \%$ & $4.6 \%$ & $3.4 \%$ & $0 \%$ \\
\hline 4 & $2.3 \%$ & $0.7 \%$ & $4.1 \%$ & $0 \%$ \\
\hline \multicolumn{5}{|l|}{ Bone lesions } \\
\hline None & $19.2 \%$ & $19.7 \%$ & $18.9 \%$ & $100 \%$ \\
\hline Minor lesions & $42.1 \%$ & $45.4 \%$ & $38.5 \%$ & $0 \%$ \\
\hline Major lesions & $38.7 \%$ & $34.9 \%$ & $42.7 \%$ & $0 \%$ \\
\hline Plasmacytoma & $11.2 \%$ & $14.1 \%$ & $8.3 \%$ & $0 \%$ \\
\hline High LDH & $15 \%$ & $16.6 \%$ & $13.3 \%$ & $5.4 \%$ \\
\hline$t(11 ; 14)$ & $13.2 \%$ & $7.4 \%$ & $18.1 \%$ & $3.4 \%$ \\
\hline $\mathrm{t}(4 ; 14)$ & $11.7 \%$ & $12.2 \%$ & $9.4 \%$ & $19.4 \%$ \\
\hline$t(14 ; 16)$ & $3 \%$ & $4.6 \%$ & $1 \%$ & $6.9 \%$ \\
\hline 17p abnormalities & $7 \%$ & $7.1 \%$ & $7.2 \%$ & $4.3 \%$ \\
\hline HR cytogenetics & $20.3 \%$ & $21.6 \%$ & $16.5 \%$ & $29.6 \%$ \\
\hline 1q gain & $47.7 \%$ & $46.4 \%$ & $50 \%$ & $26.3 \%$ \\
\hline del1p & $5.7 \%$ & $7.2 \%$ & $3 \%$ & $20 \%$ \\
\hline
\end{tabular}

Clinical features were compared distinguishing between symptomatic (transplant-candidates and noncandidates) and smoldering myeloma patients. $\mathrm{IgH}$ immunoglobulin heavy chain, IgL immunoglobulin light chain, R-ISS Revised International Staging System, ECOG Eastern Cooperative Oncology Group Performance Status, $L D H$ lactate dehydrogenase, HR cytogenetics high-risk cytogenetics.

${ }^{\text {a }}$ Proportions of each ISS group are shown instead of the R-ISS for asymptomatic myeloma patients.

rearrangements were detected, including the identification of five cases with a biallelic rearrangement, as well as one case with a potential biclonal myeloma that was later confirmed by flow cytometry (Fig. S1). Among them, 362 rearrangements $(93.3 \%)$ were productive, while 26 rearrangements (6.7\%) were unproductive (Fig. 1). Six of these unproductive sequences corresponded to light-chain myeloma patients.

\section{Gene repertoire}

$I G H V, I G H D$ and IGHJ usage was identified in productive rearrangements. Table 2 shows the frequencies of $I G H V$ gene group usage among the 362 productive rearrangements, with observed and expected frequencies (based on the number of genes per IGHV group), as well as SHM rates. No major differences were observed comparing transplant and nontransplant subgroups. 


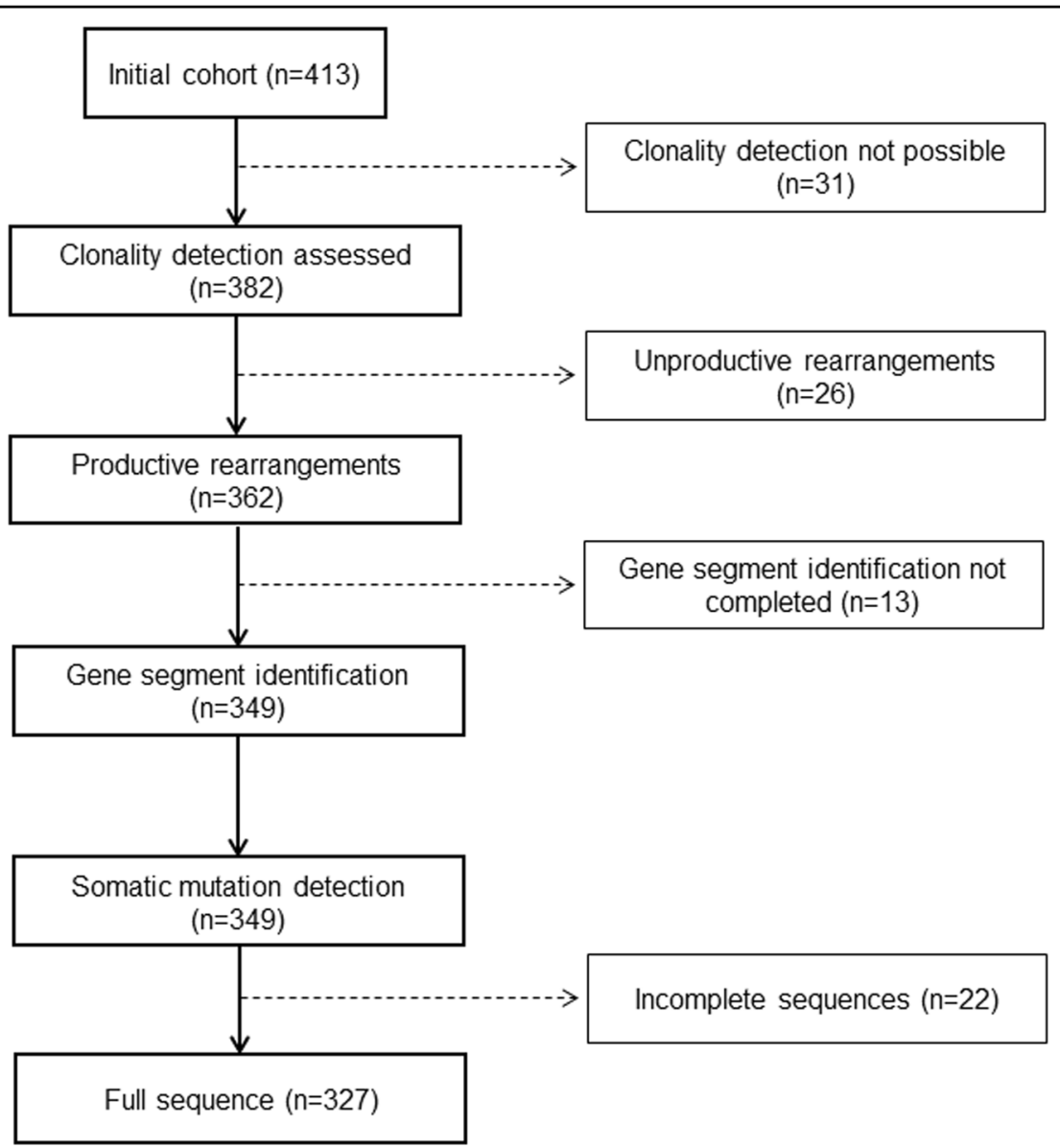

Fig. 1 Study flowchart. From the starting population $(n=413), 92$ patients were excluded. Hence, molecular profiling and survival analyses were performed with $327 / 413$ patients (79.2\%).

\section{IGHV genes}

According to the IMGT criteria $^{29,32}$, we detected 50 functional genes (Fig. S2 and Table S1) in our cohort. Five canonical IGHV genes were never seen: IGHV1-45, IGHV3-20, IGHV4-28, IGHV6-1 and IGHV7-04. Overall, IGHV3-30 was the most abundant IGHV gene, with 46/ 362 cases $(12.7 \%)$, followed by IGHV3-33 (5.2\%), IGHV323 (5\%) and IGHV1-69 (4.7\%). On the other hand, some $I G H V$ genes usually rearranged in the normal B-cell repertoire, such as IGHV3-20 or IGHV3-74, were underrepresented or totally absent. Another interesting finding was the presence of four cases using the IGHV434 gene: two of them had high SHM rates (10 and 18.9\%), both were unproductive, and patients had light-chain myeloma; the other two cases also showed higher SHM rates than the mean (20.8 and $16.7 \%$ ) and clonal rearrangements were productive. One of these patients had light-chain myeloma while the other one was IgG secretory MM.

Finally, we also found noncanonical, haplotype-dependent $I G H V$ genes in ten patients: IGHV4-30-2 $(n=1)$, IGHV4-
30-4 $(n=5)$, IGHV4-38-2 $(n=1)$ and IGHV5-10-1 $(n=3)$, and one pseudogene (IGHV3-69-1, $n=1$ ).

Compared to previous myeloma series, IGHV gene frequencies in our cohort were similar with three exceptions (IGHV3-20, IGHV4-30-2 and IGHV6-1) that were not found in our patients. We also observed that, while $I G H V 3$ gene group representation was similar between both series, IGHV3-30 was overrepresented and IGHV330-3 was underrepresented in our cohort.

\section{IGHD and IGHJ genes}

IGHD and IGHJ gene identification was assessed in 349/ 362 sequences $(96 \%)$.

IGHD3 and IGHD2 groups were the most abundant (30.4\% and 25.4\%, respectively), with IGHD3-10 (10\%) and IGHD2-21 (8\%) genes significantly overrepresented $(p<$ $0.05)$ as compared to the expected value in healthy plasma cells. Conversely, IGHD4-4 and IGHD6-25 were not found in any sequence, and all members from the IGHD1 gene group but IGHD1-26 seemed to be underrepresented (less than $1.5 \%$ each one, Fig. S3 and Table S2). All these 
Table 2 Group usage and SHM rates in IGHV genes.

\begin{tabular}{|c|c|c|c|}
\hline IGHV group & Expected frequency & Observed frequency & Median SHM \% $(95 \% \mathrm{Cl})$ \\
\hline IGHVI & $10(18.2 \%)$ & $55(15.2 \%)$ & $10.0(8.6-10.5)$ \\
\hline IGHV2 & $3(5.4 \%)$ & $31(8.6 \%)$ & $6.5(6.0-8.0)$ \\
\hline IGHV3 & $26(47.3 \%)$ & $191(52.7 \%)$ & $8.8(8.7-9.7)$ \\
\hline IGHV4 & $12(21.8 \%)$ & 67 (18.5\%) & $9.2(9.2-11.4)$ \\
\hline IGHV5 & $2(3.6 \%)$ & $18(5.0 \%)$ & $7.2(6.1-9.0)$ \\
\hline IGHV6 & $1(1.8 \%)$ & 0 & - \\
\hline IGHV7 & $1(1.8 \%)$ & 0 & - \\
\hline Total & 55 & 362 & $8.8(8.8-9.6)$ \\
\hline Comparison of SHM \% & Adjusted $p$ value & & \\
\hline IGHV1 vs. IGHV2 & 0.006 & & \\
\hline IGHV2 vs. IGHV4 & 0.001 & & \\
\hline IGHV2 vs. IGHV3 & 0.012 & & \\
\hline IGHV1 vs. IGHV5 & 0.301 & & \\
\hline IGHV4 vs. IGHV5 & 0.118 & & \\
\hline IGHV3 vs. IGHV5 & 0.790 & & \\
\hline IGHV1 vs. IGHV4 & 1 & & \\
\hline IGHV2 vs. IGHV5 & 1 & & \\
\hline IGHV3 vs. IGHV4 & 1 & & \\
\hline IGHV1 vs. IGHV3 & 1 & & \\
\hline
\end{tabular}

The first two columns show the expected IGHV gene usage if all genes were randomly selected compared with the observed frequency within our cohort, respectively. Observed and expected IGHV distributions were not different when the $\chi^{2}$ test was applied, demonstrating that there is no evidence of selection of specific gene groups in myeloma. Median somatic hypermutation rates per IGHV group are shown on the right side of the table. Below, Kruskal-Wallis-based paired comparisons are listed to determine potential differences in the hypermutation rate; a significantly lower mutation rate was found for patients using IGHV2 compared to those using IGHV1 $(p=0.006)$, IGHV3 $(p=0.012)$ and IGHV4 $(p=0.001)$.

95\% Cl 95\% confidence intervals.

features were similar in previous myeloma series and were also seen in other hematologic B-cell malignancies and in normal plasma cells, although preference for IGHD2-21 was specially marked only in myeloma.

For IGHJ gene usage, we found a deep bias in the use of certain genes matching previous observations concerning different hematologic malignancies such as CLL, MCL, and the normal bone marrow compartment: a significant overrepresentation of IGHJ4 (46.4\%) and IGHJ6 (25\%) was estimated (Fig. S4 and Table S3), while IGHJ2 and IGHJ1 were clearly underrepresented $(2.9 \%$ and $1.1 \%$, respectively). However, we noticed that, compared to previous series in myeloma, IGHJ4 was underrepresented and IGHJ6 overrepresented. In addition, IGHJI and IGHJ6 usage in healthy plasma cells looked completely different not only with respect to myeloma, but also with other mature B-cell disorders.

We explored associations between $V D J H$ repertoire and risk factors. IGHJ6 gene group was more frequent among patients with high-risk cytogenetics $(37.9 \%$ vs. $17.8 \%, p=$
0.025) considering only transplant-candidate patients. IGHD1 gene group was also more frequently used by patients harboring high-risk cytogenetics, although this association was restricted to patients not candidates for transplantation $(41.7 \%$ vs. $13.2 \%, p=0.026)$. However, there were not relevant associations between the usage of these genes and the outcome in terms of PFS or OS.

Transplant-eligible and -ineligible patients showed similar proportions in $V D J H$ gene usage (Table S4). In the same line, high-risk smoldering patients did not show significant differences in the use of $V D J H$ genes compared to symptomatic patients.

\section{Somatic hypermutation rates and IGH composition}

Somatic mutations could be correctly identified in 349 sequences: mutational load was high (mean: $9.2 \% \pm$ 3.8; median: $8.8 \%$; 95\% CI: $8.8-9.6 \%$; range: $0-22.7 \%$ ), with five cases showing more than $98 \%$ of homology with germline genes. Comparing SHM rates between IGHV groups, we found a significantly lower rate for IGHV2 
compared to IGHV1, IGHV3 and IGHV4 (Table 2). Median SHM ratios of transplant-eligible and -ineligible patients were similar $(9.0 \%$ vs. $8.5 \%$ respectively, $p=$ $0.869)$. Median SHM rate in symptomatic and asymptomatic patients was also similar $(9.0 \%$ vs. $8.8 \%, p=0.845)$. The complete sequence was available in $327 / 362$ cases, so we continued the analysis with this set (Fig. 1).

As far as the junction region was concerned, nucleotide addition appeared in $92.5 \%$ of cases for the $\mathrm{N} 1$ region, and in $88.6 \%$ of cases for the N2 region. Only three cases did not show evidence of nucleotide addition, either in $\mathrm{N} 1$ or in N2. Mean Guanine/Cytosine content of the N-regions was $58.8 \%$. Evidence of exonuclease activity in at least one of the two junction regions (IGHV-to-IGHD and/or IGHDto-IGHJ) was observed in all rearrangements, although $32.2 \%$ of cases displayed a fully conserved $I G H V-3^{\prime}$ edge.

\section{CDR3 amino acid composition}

According to the IMGT numbering, we identified CDR3 positions (from amino acid 105 to amino acid 117). Median CDR3 length was 15 amino acids (range 6-29); this length was consistent across different myeloma subgroups (symptomatic, asymptomatic, transplant-eligible and transplant-ineligible patients). Amino acid proportions in myeloma were not significantly different from the normal B-cell population (Fig. S5), with predominance of Glycine, Alanine, Aspartic acid and Tyrosine.

\section{Clustering analysis}

CDR3 sequences from productive rearrangements $(n=$ 362 ) were subjected to cluster analysis. We found one pair from our cohort that showed more than $60 \%$ of amino acid similarity. Consequently, and given they were clusters not previously described, we applied the secondary criteria. Although CDR3 lengths were similar (13 vs. 15 amino acids) and both rearrangements displayed the same $I G H J 4 * 02$ germinal gene, IGHV and IGHD groups, as well as $I G H D$ reading frames, were not concordant. Thus, we could not find any stereotyped immunoglobulin cluster among these myeloma patients.

\section{Impact of molecular variables on clinical outcomes}

Considering patients with symptomatic myeloma, after a median follow-up of 7.6 years (range 0.5-24.1), 120 patients had died; the majority of them $(n=76)$ were transplant-ineligible. ROC curves showed that 7 and $8 \%$ were the most optimal cutoffs for prognostic value of SHM; optimal cutoffs for serum albumin $(3.2 \mathrm{~g} / \mathrm{dL}), \beta 2$ microglobulin $(3.5 \mathrm{mg} / \mathrm{dL})$, hemoglobin $(9 \mathrm{~g} / \mathrm{dL})$ and creatinine $(1 \mathrm{mg} / \mathrm{dL})$ were also estimated. In addition, IGHD2 and IGHD3 users were grouped for subsequent analyses, because they were the most common IGHD groups and this bias has been observed in nearly every Bcell malignancy ${ }^{9-18}$. Finally, all molecular data described in previous paragraphs were combined with clinical data to perform statistical comparisons in transplant-eligible and -ineligible patients.

We first looked for relationships between treatment response and molecular variables. No major associations were seen, but two exceptions: (1) SHM $\geq 7 \%$ associated with a higher rate of $\mathrm{CR} / \mathrm{sCR}$ compared to patients with $\mathrm{SHM}<7 \%$ in the nontransplant subset $(45.8 \%$ vs. $27.3 \%$, respectively; $p=0.026$ ), and (2) IGHD3-3 usage was associated with a better response in the transplant subgroup $(n=10)$, since $80 \%$ of MM patients with an IGH rearrangement choosing this particular gene achieved CR/ sCR vs. $36.8 \%$ for other IGHD genes $(p=0.019)$.

In addition, we detected that SHM rates were progressively higher when symptomatic patients were analyzed based on the differentiation stage of their pathologic plasma cells: median SHM levels for CD19+/CD81+ (immature), CD19-/CD81+ (intermediate) and CD19 -/CD81- (mature) plasma-cell subsets were 7.0\% (95\% CI: $5.2-11.4 \%), 8.50 \%$ (95\% CI: 7.9-9.6\%), and 9.3\% (95\% CI: $9.1-10.2 \%)$, respectively $(p=0.029)$. Moreover, median PFS increased from intermediate to mature subgroups (35.4 vs. 64.0 months respectively, $p=0.024$ ) but not from immature to intermediate subsets, probably because we only found 11 CD19+/CD81+ cases in our series.

\section{Transplant-ineligible patients}

In the univariate analysis for PFS, improved survival of transplant-ineligible patients was associated with $0-1$ ECOG stages $(p=0.003), \mathrm{CR} / \mathrm{sCR}$ after the end of induction $\left(p=2 \times 10^{-6}\right)$, baseline hemoglobin $\geq 9 \mathrm{~g} / \mathrm{dL} \quad(p=$ $0.002)$, no use of the IGHD4 gene group $(p=0.036)$ (Fig. 2a) or specifically the IGHD4-11 gene $(p=0.0003)$, use of the IGHD2/IGHD3 gene groups $(p=0.012)$ (Fig. $2 \mathrm{~b})$, and SHM rate $\geq 7 \%(p=0.006)$ (Fig. 3a). Improved OS rates were observed in the univariate analysis for those patients with $\mathrm{CR} / \mathrm{sCR}$ after induction ( $p=0.00026$ ), SHM rate $\geq 7 \%$ $(p=0.00024)$ (Fig. 3b), standard-risk cytogenetics $(p=$ $0.031)$ and serum hemoglobin $\geq 9 \mathrm{~g} / \mathrm{dL}(p=0.002)$.

One hundred and eleven transplant-ineligible patients were included in the multivariate analysis. For PFS (Table 3), CR/sCR achievement $\left(p=10^{-6}\right.$, HR: 0.311, 95\% CI: $0.196-0.494)$ and the use of IGHD2/IGHD3 gene groups $(p=0.006$, HR: $0.552,95 \%$ CI: 0.361 -0.845 ) appeared as independent prognostic factors of prolonged survival rates. For OS, CR achievement after induction retained its independent prognostic value for improved survival probability ( $p=0.010$, HR: $0.347,95 \%$ CI: $0.154-0.780)$ as well as SHM $\geq 7 \%(p=0.001$, HR: 0.291, 95\% CI: $0.137-0.618)$ and standard cytogenetic risk $(p=0.016$, HR: 0.369 , 95\% CI: 0.164-0.832). Moreover, SHM rate was included in the multivariate regression model not only as a discrete variable but also as a continuous one to avoid potential bias induced with 

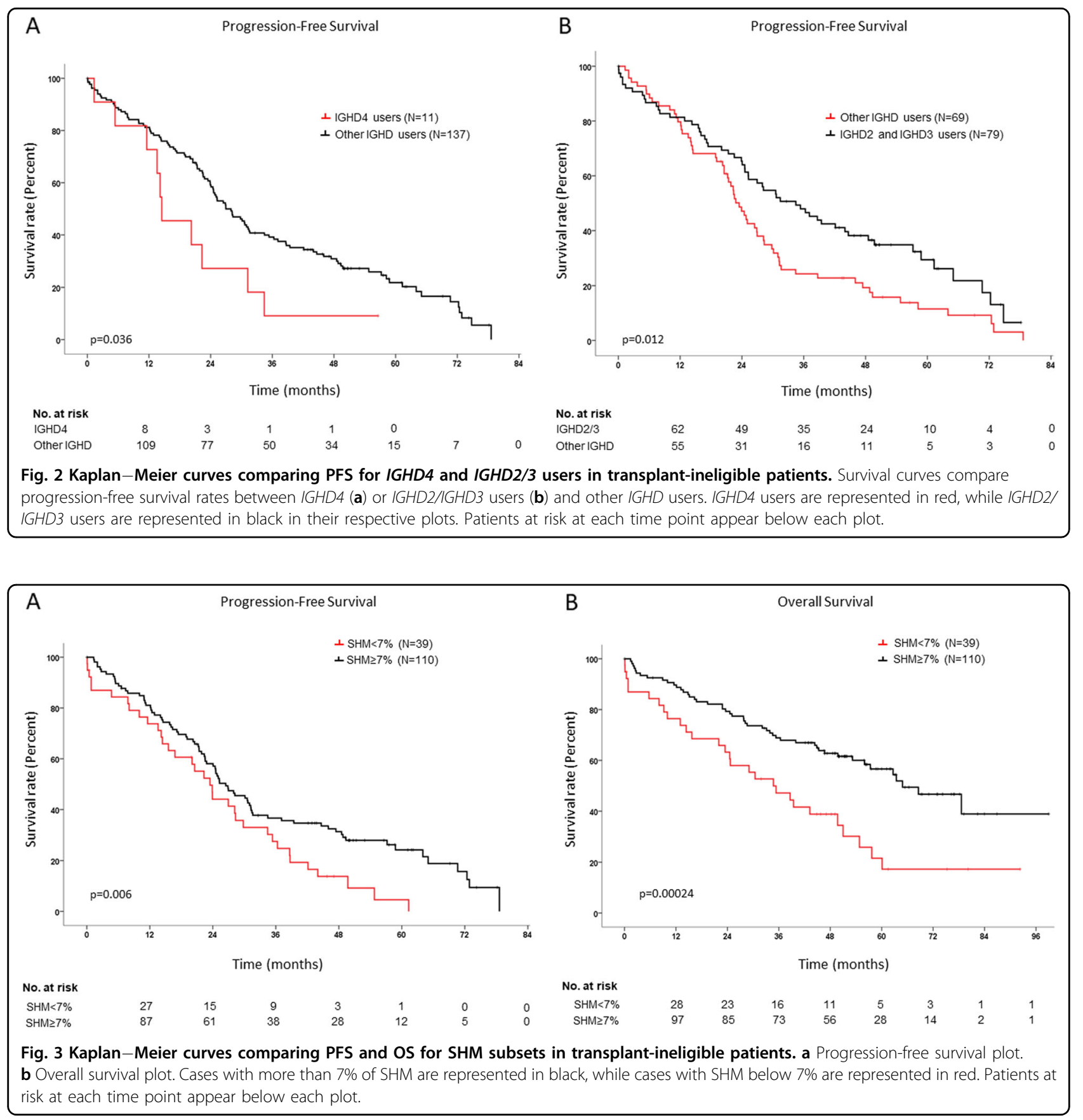

the cutoff selection of 7\%; in this case, the variable retained its prognostic value ( $p=0.017$, HR: $0.909,95 \%$ CI: $0.840-0.983$ ) along with achieving CR/sCR and the absence of cytogenetic risk, showing that an increment of $1 \%$ in SHM reduces death risk by $9 \%$.

\section{Transplant-eligible patients}

Univariate analysis for PFS showed improved outcomes for patients in R-ISS stage I vs. II $(p=0.002)$ and III $(p=$ $0.004)$, standard-risk cytogenetics $\left(p=3 \times 10^{-6}\right), 0-1$ ECOG stages $(p=0.015)$, absence of 1q gains $(p=0.019)$ and serum $\beta 2$ microglobulin $<3.5 \mathrm{mg} / \mathrm{dL}(p=0.023)$. The R-ISS stage $\left(p=6 \times 10^{-6}\right)$, high-risk cytogenetics $(p=4 \times$ $\left.10^{-6}\right)$, the ECOG score $(p=0.004), 1 \mathrm{q}$ gains $(p=0.016)$ and serum $\beta 2$ microglobulin $(p=0.002)$ also showed significant associations with the OS in the same direction as PFS. Interestingly, no molecular variable was significantly associated with survival in this subset of patients.

One hundred and thirty-seven transplant-eligible patients were included in the multivariate analysis. Presence of 1q gains ( $p=0.046$, HR: $2.187,95 \% \mathrm{CI}$ : $1.015-4.714)$ and high-risk cytogenetics $(p=$ 
Table 3 Univariate and multivariate analysis of clinical and biological factors influencing PFS and OS of transplantineligible patients in our cohort.

\begin{tabular}{|c|c|c|c|c|c|c|c|c|}
\hline \multirow[t]{2}{*}{ Variable } & \multicolumn{2}{|c|}{ Univariate analysis for PFS } & \multicolumn{2}{|c|}{ Multivariate analysis for PFS } & \multicolumn{2}{|c|}{ Univariate analysis for OS } & \multicolumn{2}{|c|}{ Multivariate analysis for OS } \\
\hline & Median survival & $p$ & $\mathrm{HR}[95 \% \mathrm{Cl}]$ & $p$ & Median survival & $p$ & $\mathrm{HR}[95 \% \mathrm{Cl}]$ & $p$ \\
\hline \multicolumn{9}{|l|}{ ECOG score } \\
\hline $0-1$ & 26.94 & 0.003 & & NS & & NS & & \\
\hline $2-4$ & 21.35 & & & & & & & \\
\hline \multicolumn{9}{|c|}{ Serum hemoglobin } \\
\hline$<9 \mathrm{~g} / \mathrm{dL}$ & 14.03 & 0.001 & & NS & 24.54 & 0.002 & & NS \\
\hline$\geq 9 \mathrm{~g} / \mathrm{dL}$ & 28.32 & & & & 64.85 & & & \\
\hline \multicolumn{9}{|c|}{ Post induction response } \\
\hline $\mathrm{sCR} / \mathrm{CR}$ & 49.35 & 0.008 & $0.311[0.196-0.494]$ & $1 \mathrm{E}-05$ & Not reached & 0.00026 & $0.347[0.154-0.780]$ & 0.010 \\
\hline VGPR/PR & 23.88 & & & & 53.19 & & & \\
\hline \multicolumn{9}{|l|}{ Cytogenetic risk } \\
\hline High risk & 36.30 & 0.196 & & NS & 78.59 & 0.031 & $0.369[0.164-0.832]$ & 0.016 \\
\hline Standard risk & 25.03 & & & & 39.95 & & & \\
\hline \multicolumn{9}{|l|}{ |GHD4 usage } \\
\hline Yes & 14.49 & 0.036 & & NS & & NS & & \\
\hline No & 27.96 & & & & & & & \\
\hline \multicolumn{9}{|l|}{ |GHD4-11 usage } \\
\hline Yes & 14.16 & 0.0003 & & NS & & NS & & \\
\hline No & 26.94 & & & & & & & \\
\hline \multicolumn{9}{|c|}{ IGHD2 and IGHD3 usage } \\
\hline Yes & 34.46 & 0.012 & $0.552[0.361-0.845]$ & 0.006 & & NS & & \\
\hline No & 23.46 & & & & & & & \\
\hline \multicolumn{9}{|l|}{ SHM level } \\
\hline$<7 \%$ & 23.46 & 0.006 & & NS & 34.73 & 0.00024 & $0.291[0.137-0.618]$ & 0.001 \\
\hline$\geq 7 \%$ & 26.45 & & & & 64.85 & & & \\
\hline
\end{tabular}

Univariate analysis was first performed to evaluate the role of each clinical and biological variable described in this study. The table only shows statistically significant variables that were later included in the multivariate analysis. Median survival is shown in months.

$H R$ hazard ratio, $\mathrm{Cl}$ confidence interval, NS nonsignificant.

0.000021, HR: 4.690, 95\% CI: 2.267-9.700) were associated with lower PFS rates. High-risk cytogenetics retained its independent prognostic value for shorter OS $(p=0.001$, HR: $8.030,95 \%$ CI: $2.406-26.809)$, while serum $\beta 2$ microglobulin levels $<3.5 \mathrm{mg} / \mathrm{dL}$ associated with better outcomes ( $p=0.039$, HR: 0.116, 95\% CI: 0.015-0.899).

\section{Discussion}

Here, we analyzed in depth the $I G H$ locus, trying to correlate its characteristics with clinical data in a large cohort of $413 \mathrm{MM}$ patients included in clinical trials. $V D J H$ usage description provided new insights into the clonal differentiation and possible clinical implications for MM, that could potentially improve patient care.

$I G H V$ gene repertoire reflected its normal counterpart, with $I G H V 3$ being the predominant gene group. As in other series, IGHV3 was overrepresented and IGHVI underrepresented, although differences were not significant, with a much less pronounced bias than that observed in CLL ${ }^{12,13,31}$. Similarly, IGHV3-30, IGHV4-59 and IGHV439 were overrepresented with respect to other IGHV genes $^{34-36}$, and although these genes are also common in other lymphoproliferative disorders and normal plasma cells, we noted that IGHV3-30 was specifically selected only in myeloma. In contrast, IGHV3-23 and IGHV1-69 appeared to be less frequently selected in $\mathrm{MM}(\geq 8 \%$ of representation in the normal B-cell population ${ }^{35,36}$ and Bcell pathologies vs. $\leq 5 \%$ in our series).

IGHV4-34 representation is high in hematologic malignancies such as B-ALL, CLL, MCL or diffuse large B-cell lymphoma as well as in normal B cells ${ }^{9,11,31}$. Interestingly, this gene is usually absent in the normal plasma-cell population ${ }^{37}$, although it shows a wide interindividual usage frequency ${ }^{38}$, and it is rarely selected in $\mathrm{MM}^{17}$, but in this work it was not completely absent. IGHV4-34 has been characterized as an inherently autoreactive antibody ${ }^{39,40}$ for it is widely represented in autoimmune diseases (i.e. systemic lupus erythematosus). Here, we found four clonal IGHV4-34 rearrangements; they showed very high SHM rates and were usually nonproductive, leading to light-chain myelomas. This could be explained by the fact that during plasma-cell differentiation, those cells displaying autoreactive-prone 
genes within their IGH rearrangements would be specially targeted by the somatic hypermutation machinery, trying to make them able to recognize only foreign antigens. Moreover, in our series, two out of the three unproductive rearrangements using VH1-69, another gene commonly associated with autoimmune events, such as Thrombotic Thrombocytopenic Purpura ${ }^{41}$, and highly represented in CLL, were light-chain myelomas with some of the highest SHM rates in our cohort (mean 12.23\%). Interestingly, this gene is commonly unmutated in CLL patients ${ }^{42,43}$.

SHM level was high and similar to what has been observed in other studies for healthy and pathological plasma and memory B cells. This would be in line with the post-germinal center origin of myeloma ${ }^{8}$. A striking finding was the presence of five $\mathrm{MM}$ cases with no apparent SHM; however, the $I G H V$ region could not be completely sequenced (the entire FWR1 and part of the FWR2 was missing); from our experience, we think the mutational load was most likely underestimated, rather than being truly unmutated cases.

CDR3 composition resembled the normal immunoglobulin repertoire, with similar length, amino acid use, or evidence of $\mathrm{TdT}$ and exonuclease activity ${ }^{44}$. This composition diverges from CLL, MCL or MZL patterns, where restricted antibody sets account for approximately 30\% of cases $^{45-47}$. Accordingly, cluster analysis failed to demonstrate the presence of stereotyped receptors in our cohort, as it has been shown before in myeloma patients ${ }^{48}$. Overall, the gene repertoire in our series was very close to previous reports $^{17,18,48}$, although functional $I G H V$ and $I G H D$ genes, whose use has not been reported in myeloma to date, have been found in this cohort probably due to its larger size. On the contrary, other genes such as IGHV1-18, IGHV3-20, IGHV4-30-2 and IGHV6-1, found in Italian ${ }^{48}$ or Greek ${ }^{18}$ patients, are absent in Spanish patients, maybe reflecting differences in the normal repertoire between different Mediterranean subpopulations. No differences were observed in the median length of the CDR3 region, and somatic hypermutation was slightly higher than in previous publications; however, this variation was not statistically significant.

The most remarkable finding was the association between a higher SHM level and an improved survival rate. The explanation for this situation seems to be connected with the maturation stage of tumor cells, represented by the expression of CD19 and CD81 biomarkers, which is consistent with previous findings regarding the immune profile of tumor plasma cells: ${ }^{49}$ it suggests that early genetic alterations in the ontogeny of germinal-center B cells would provide a more aggressive profile in MM, which would be strong enough to overcome SHM and/or CSR earlier. Conversely, the acquisition of late, more harmless genetic events would allow tumor B cells to undergo SHM and CSR processes and this would lead to better outcomes. Another possible explanation is related to the proliferation ratio of tumor cells: highly proliferative B cells (and therefore, more aggressive clones) activate DNA repair pathways that are able to reduce the SHM level, as it has been shown in CLL patients $^{50,51}$. However, the SHM cutoff is based on FR1 primers; this threshold could differ when using leader primers.

Another striking feature was the correlation between the use of IGHD2 and IGHD3 gene groups and the outcome. An increased usage of these particular groups has also been observed in other series suggesting a positive selective pressure over them ${ }^{8,17,18}$, since the bias was observed only for complete rearrangements. Nevertheless, whether or not the relationship between IGHD usage and survival is a myeloma characteristic remains uncertain.

SHM and IGHD usage were associated with the outcome only in the elderly, transplant-ineligible patients. Thereby, the effect of intensive regimens could be the main reason to explain why the negative prognostic impact of these molecular characteristics is diluted in younger, fit patients.

Regarding the value of clinical variables on the patient outcome, the prognostic role of cytogenetics, hemoglobin, albumin, $\beta 2$ microglobulin and ECOG in MM was confirmed $^{52}$. Cytogenetic risk has to be mentioned as this variable showed significant associations with survival in all cases but when applied to PFS in transplant-ineligible patients. The absence of FISH studies for most patients diagnosed from 1995 to 2000 (most of them being older) made possible to find only 17 patients with high-risk cytogenetics in this subgroup, which could explain this result.

In conclusion, we have shown that multiple myeloma plasma cells resemble the normal mature B-cell repertoire but, in contrast to CLL, MCL or MZL, stereotyped receptors were absent ${ }^{14,15,53,54}$. Light-chain and nonsecretory myeloma cases could be explained by incorrect $I G H$ rearrangements at the genomic DNA level, but not always, which means that other effects could hamper the immunoglobulin production, including transcriptional or translational defects. Finally, here, and for the first time, we have reported how some molecular characteristics of the IGH gene rearrangement, such as higher SHM rates or IGHD2/IGHD3 gene usage, could positively impact on the outcome of patients. A validation series is currently planned to elucidate whether these findings could be applicable in real-life patients; if validated in independent studies, they could be considered as new molecular markers for PFS and OS in multiple myeloma.

\footnotetext{
Acknowledgements

This work was partially supported by the Instituto de Salud Carlos III (ISCIII) Spanish Ministry of Economy and Competitiveness PI15/01956, CIBERONCCB16/12/00233, and "Una manera de hacer Europa" (Innocampus; CEI-20101-0010)". M.G.-A., I.P.-C., and C.J. are supported by the Fundación Española
} 
de Hematología y Hemoterapia (FEHH, co-funded by Fundación Cris in the latter case), A.M. by the European Social Fund and the Spanish Education Council through the University of Salamanca, and M.E.S. by the ISCIII (CPII18/ 00028). All Spanish funding is co-sponsored by the European Union FEDER program. The authors wish to thank José Pérez, Alicia Antón, Montserrat Hernández-Ruano, Estrella Arnés, Rebeca Maldonado, Mercedes Jiménez, Alejandra Martín, Isabel Sánchez, Rocío Corral and Francisco Boix (University Hospital of Salamanca, Spain) for their technical support, as well as the Spanish Multiple Myeloma "GEM-PETHEMA" group for providing clinical data. The authors are also very grateful to the patients who participated in this study.

\section{Author details}

'Hospital Universitario de Salamanca (HUSAL), IBSAL, IBMCC (USAL-CSIC), CIBERONC, Salamanca, Spain. ${ }^{2}$ Hospital 12 de Octubre, CIBERONC, Madrid, Spain. ${ }^{3}$ Complejo Hospitalario, León, Spain. ${ }^{4}$ Hospital Vall d'Hebrón, Barcelona, Spain. ${ }^{5}$ Hospital Universitario de Canarias, La Laguna, Spain. ${ }^{6}$ Hospital Duran i Reynals, Institut Català d'Oncología (ICO), L'Hospitalet de Llobregat, Barcelona, Spain. ${ }^{7}$ Hospital Germans Trias i Pujol, Institut Català d'Oncología (ICO), Institut Josep Carreras, Badalona, Spain. ${ }^{8}$ Hospital Clínic i Provincial, Institut de Investicacions Biomediques August Pi i Sunyer (IDIBAPS), Barcelona, Spain. ${ }^{9}$ Clínica Universidad de Navarra (CUN), Centro de Investigación Médica Aplicada, IDISNA, CIBERONC, Pamplona, Spain

\section{Author contributions}

A.M., R.G.-S. and M. González conceived and designed the study. NP, FE, NCG, M. González, M.T.H., A.S., A.O., M.-J.C., J.M.-L., J.B., J.-J.L., M. Gironella, J.F. S.M., M.-V.M. and R.G.-S. recruited patients for the Spanish Multiple Myeloma Working Group (GEM-PETHEMA) clinical trials. A.M., C.J., M.E.S., I.P.-C., M.G.-A. and A.B. produced the molecular results. A.M., M.E.S., V.G.-C. and R.G.-S. analysed the data, performed the statistical analyses, and wrote the first draft of the manuscript. M.A., N.P., I.P.-C., M.G.-A., V.G.-C. and R.G.-S. provided support for statistical analyses and clinical data interpretation. M.E.S., N.P., M.A., V.G.-C., F.E., N.C.G., M. González, M.T.H., A.S., A.O., M.-J.C., J.M.-L., J.B., J.-J.L., M. Gironella, J.F.S.M., M.-V.M. and R.G.-S. reviewed the final manuscript. R.G.-S. and M. González obtained funding for the study. R.G.-S., the head of the group, produced the final revision of the manuscript and gave final approval for its publication.

\section{Conflict of interest}

Authors report grants PI15/01956, CIBERONC-CB16/12/00233, and CEl-2010-10010 during the conduct of the study. A.M., M.G.-A., I.P.-C., C.J., V.G.-C. and M.E.S. are or were supported by the Spanish government. Authors have received honoraria from, consulted or served in an advisory role for, or are on the board of directors of Janssen (N.P., F.E., A.O., J.B., J.-J.L., J.F.S.M., M.-V.M., J.M.-L., R.G.-S.), Takeda (N.P., A.O., J.F.S.M., M.V.M., R.G.-S.), Celgene (N.P., A.O., J.-J.L., J.F.S.M., M.-V.M.), Amgen (N.P., J.-J.L., J.F.S.M., M.-V.M), Bristol-Myers Squibb (J.F.S.M., M.-V.M., J.M.-L., R.G.-S.), Novartis (J.F.S.M., J.M.-L.), MSD (J.F.S.M.), Sanofi (J.F.S.M.), Roche (J.F.S.M.), Hospira (R.G.S.), Pharmacyclics (R.G.-S.), Gilead (R.G.-S.) and Incyte (R.G.S.S.). The remaining authors have no relevant conflict of interest to disclose.

\section{Publisher's note}

Springer Nature remains neutral with regard to jurisdictional claims in published maps and institutional affiliations.

Supplementary Information accompanies this paper at (https://doi.org/ 10.1038/s41408-020-0283-8).

Received: 4 September 2019 Revised: 16 January 2020 Accepted: 21 January 2020

Published online: 06 February 2020

\section{References}

1. Early, P., Huang, H., Davis, M., Calame, K. \& Hood, L. An immunoglobulin heavy chain variable region gene is generated from three segments of DNA: $\mathrm{VH}, \mathrm{D}$ and JH. Cell 19, 981-992 (1980).
2. Oettinger, M. A., Schatz, D. G., Gorka, C. \& Baltimore, D. RAG-1 and RAG-2, adjacent genes that synergistically activate $V(D) J$ recombination. Science $\mathbf{2 4 8}$, 1517-1523 (1990).

3. Komori, T., Okada, A., Stewart, V. \& Alt, F. W. Lack of $\mathrm{N}$ regions in antigen receptor variable region genes of TdT-deficient lymphocytes. Science 261, 1171-1175 (1993).

4. Matsuda, F. et al. The complete nucleotide sequence of the human immunoglobulin heavy chain variable region locus. J. Exp. Med. 188, 2151-2162 (1998).

5. Tonegawa, S. Somatic generation of antibody diversity. Nature $\mathbf{3 0 2}, \mathbf{5 7 5 - 5 8 1}$ (1983).

6. Mostoslavsky, R., Alt, F. W. \& Rajewsky, K. The lingering enigma of the allelic exclusion mechanism. Cell 118, 539-544 (2004).

7. Honjo, T., Kinoshita, K. \& Muramatsu, M. Molecular mechanism of class switch recombination: linkage with somatic hypermutation. Annu. Rev. Immunol. 20, 165-196 (2002).

8. González, D. et al. Immunoglobulin gene rearrangements and the pathogenesis of multiple myeloma. Blood 110, 3112-3121 (2007).

9. Hockley, S. L. et al. The prognostic impact of clinical and molecular features in hairy cell leukaemia variant and splenic marginal zone lymphoma. Br. J. Haematol. 158, 347-354 (2012).

10. Petrikkos, L. et al. Clonotypic analysis of immunoglobulin heavy chain sequences in patients with Waldenström's macroglobulinemia: correlation with MYD88 L265P somatic mutation status, clinical features, and outcome. BioMed. Res. Int. 2014, 809103 (2014).

11. Mroczek, E. S. et al. Differences in the composition of the human antibody repertoire by B cell subsets in the blood. Front. Immunol. 5, 96 (2014).

12. Marinelli, M. et al. Immunoglobulin gene rearrangements in Chinese and Italian patients with chronic lymphocytic leukemia. Oncotarget 7, 20520-20531 (2016).

13. Hamblin, T. J., Davis, Z., Gardiner, A., Oscier, D. G. \& Stevenson, F. K. Unmutated Ig $V(H)$ genes are associated with a more aggressive form of chronic lymphocytic leukemia. Blood 94, 1848-1854 (1999).

14. Agathangelidis, A. et al. Stereotyped B-cell receptors in one-third of chronic lymphocytic leukemia: a molecular classification with implications for targeted therapies. Blood 119, 4467-4475 (2012).

15. Darzentas, N. \& Stamatopoulos, K. Stereotyped B cell receptors in B cell leukemias and lymphomas. Methods Mol. Biol. 971, 135-148 (2013).

16. Kiyoi, H., Naito, K., Ohno, R. \& Naoe, T. Comparable gene structure of the immunoglobulin heavy chain variable region between multiple myeloma and normal bone marrow lymphocytes. Leukemia 10, 1804-1812 (1996).

17. González, D. et al. Molecular characteristics and gene segment usage in IGH gene rearrangements in multiple myeloma. Haematologica 90, 906-913 (2005).

18. Hadzidimitriou, A. et al. Immunoglobulin genes in multiple myeloma: expressed and non-expressed repertoires, heavy and light chain pairings and somatic mutation patterns in a series of 101 cases. Haematologica 91, 781-787 (2006).

19. Lahuerta, J. J. et al. Busulfan $12 \mathrm{mg} / \mathrm{kg}$ plus melphalan $140 \mathrm{mg} / \mathrm{m} 2$ versus melphalan $200 \mathrm{mg} / \mathrm{m} 2$ as conditioning regimens for autologous transplantation in newly diagnosed multiple myeloma patients included in the PETHEMAVGEM2000 study. Haematologica 95, 1913-1920 (2010).

20. Mateos, M. V. et al. Bortezomib, melphalan, and prednisone versus bortezomib, thalidomide, and prednisone as induction therapy followed by maintenance treatment with bortezomib and thalidomide versus bortezomib and prednisone in elderly patients with untreated multiple myeloma: a randomised trial. Lancet Oncol. 11, 934-941 (2010).

21. Rosinol, L. et al. Superiority of bortezomib, thalidomide, and dexamethasone (VTD) as induction pretransplantation therapy in multiple myeloma: a randomized phase 3 PETHEMAVGEM study. Blood 120, 1589-1596 (2012).

22. Mateos, M. V. et al. Sequential vs alternating administration of VMP and Rd in elderly patients with newly diagnosed MM. Blood 127, 420-425 (2016).

23. Mateos, M. V. et al. Lenalidomide plus dexamethasone versus observation in patients with high-risk smouldering multiple myeloma (QuiRedex): long-term follow-up of a randomised, controlled, phase 3 trial. Lancet Oncol. 17, 1127-1136 (2016).

24. Rosinol, L. et al. Bortezomib, lenalidomide, and dexamethasone as induction therapy prior to autologous transplant in multiple myeloma. Blood 134, 1337-1345 (2019).

25. Mateos, M. V. et al. Lenalidomide plus dexamethasone for high-risk smoldering multiple myeloma. N. Engl. J. Med. 369, 438-447 (2013). 
26. Chng, W. J. et al. IMWG consensus on risk stratification in multiple myeloma. Leukemia 28, 269-277 (2014).

27. Palumbo, A. et al. Revised International Staging System for multiple myeloma: a report from International Myeloma Working Group. J. Clin. Oncol. 33 2863-2869 (2015).

28. van Dongen, J. J. M. et al. Design and standardization of PCR primers and protocols for detection of clonal immunoglobulin and T-cell receptor gene recombinations in suspect lymphoproliferations: report of the BIOMED-2 Concerted Action BMH4CT98-3936. Leukemia 17, 2257-2317 (2003).

29. Brochet, X., Lefranc, M.-P. \& Giudicelli, V. IMGTN-QUEST: the highly customized and integrated system for IG and TR standardized $V$-J and V-D-J sequence analysis. Nucleic Acids Res. 36, W503-W508 (2008).

30. Larkin, M. A. et al. Clustal $W$ and Clustal $X$ version 2.0. Bioinformatics. 23, 2947-2948 (2007).

31. Stamatopoulos, K. et al. Over $20 \%$ of patients with chronic lymphocytic leukemia carry stereotyped receptors: pathogenetic implications and clinical correlations. Blood. 109, 259-270 (2007)

32. Lefranc, M. P. et al. IMGT, the international ImMunoGeneTics information system. Nucleic Acids Res. 37, D1006-D1012 (2009).

33. Messmer, B. T. et al. Multiple distinct sets of stereotyped antigen receptors indicate a role for antigen in promoting chronic lymphocytic leukemia. J. Exp. Med. 200, 519-525 (2004).

34. Kosmas, C. et al. Origin and diversification of the clonogenic cell in multiple myeloma: lessons from the immunoglobulin repertoire. Leukemia $\mathbf{1 4}$ 1718-1726 (2000).

35. Brezinschek, H. P. et al. Analysis of the human $\mathrm{VH}$ gene repertoire. Differential effects of selection and somatic hypermutation on human peripheral CD5(+)/lgM+ and CD5(-)/lgM+ B cells. J. Clin. Invest. 99, 2488-2501 (1997).

36. Kraj, P. et al. The human heavy chain lg $\vee$ region gene repertoire is biased at all stages of B cell ontogeny, including early pre-B cells. J. Immunol. 158, 5824-5832 (1997).

37. Pugh-Bernard, A. E. et al. Regulation of inherently autoreactive VH434 B cells in the maintenance of human B cell tolerance. J. Clin. Invest. 108, 1061-1070 (2001).

38. Turchaninova, M. A. et al. High-quality full-length immunoglobulin profiling with unique molecular barcoding. Nat. Protoc. 11, 1599-1616 (2016).

39. Bhat, N. M., Lee, L. M., van Vollenhoven, R. F., Teng, N. N. H. \& Bieber, M. M. VH434 encoded antibody in systemic lupus erythematosus: effect of isotype. $J$. Rheumatol. 29, 2114-2121 (2002).

40. Mockridge, C. I. et al. Common patterns of B cell perturbation and expanded V4-34 immunoglobulin gene usage in autoimmunity and infection. Autoimmunity 37, 9-15 (2004).
41. Pos, W. et al. VH1-69 germline encoded antibodies directed towards ADAMTS13 in patients with acquired thrombotic thrombocytopenic purpura. J. Thromb. Haemost. 7, 421-428 (2009).

42. Duke, $\mathrm{V}$. M. et al. $\mathrm{V}(\mathrm{H})$ gene usage differs in germline and mutated Bcell chronic lymphocytic leukemia. Haematologica. 88, 1259-1271 (2003).

43. González-Gascón, Y. et al. Mutation status and immunoglobulin gene rearrangements in patients from northwest and central region of Spain with chronic lymphocytic leukemia. BioMed. Res. Int. 2014, 257517 (2014).

44. Shi, B. et al. Comparative analysis of human and mouse immunoglobulin variable heavy regions from IMGT/LIGM-DB with IMGT/HighV-QUEST. Theor. Biol. Med. Model. 11, 30 (2014)

45. Darzentas, N. et al. A different ontogenesis for chronic lymphocytic leukemia cases carrying stereotyped antigen receptors: molecular and computational evidence. Leukemia 24, 125-132 (2010).

46. Agathangelidis, A., Hadzidimitriou, A., Rosenquist, R. \& Stamatopoulos, K. Unlocking the secrets of immunoglobulin receptors in mantle cell lymphoma: implications for the origin and selection of the malignant cells. Semin. Cancer Biol. 21, 299-307 (2011)

47. Hadzidimitriou, A. et al. Is there a role for antigen selection in mantle cell lymphoma? Immunogenetic support from a series of 807 cases. Blood $\mathbf{1 1 8}$ 3088-3095 (2011).

48. Ferrero, S. et al. Multiple myeloma shows no intra-disease clustering of immunoglobulin heavy chain genes. Haematologica 97, 849-853 (2012).

49. Paiva, B. et al. Differentiation stage of mieloma plasma cells: biological and clinical significance. Leukemia 31, 382-392 (2017).

50. van Gent, R. et al. In vivo dynamics of stable chronic lymphocytic leukemia inversely correlate with somatic hypermutation levels and suggest no major leukemic turnover in bone marrow. Cancer Res. 68, 10137-10144 (2008).

51. Giné, E. et al. Expanded and highly active proliferation centers identify a histological subtype of chronic lymphocytic leukemia ("accelerated" chronic lymphocytic leukemia) with aggressive clinical behavior. Haematologica 95 1526-1533 (2010)

52. Rajkumar, S. V. Multiple myeloma: 2016 update on diagnosis, risk-stratification, and management. Am. J. Hematol. 91, 719-734 (2016).

53. Varettoni, M. et al. Clues to pathogenesis of Waldenström macroglobulinemia and immunoglobulin $\mathrm{M}$ monoclonal gammopathy of undetermined significance provided by analysis of immunoglobulin heavy chain gene rearrangement and clustering of B-cell receptors. Leuk. Lymphoma 54, 2485-2489 (2013).

54. Zibellini, S. et al. Stereotyped patterns of B-cell receptor in splenic marginal zone lymphoma. Haematologica 95, 1792-1796 (2010). 